\title{
DENSE SETS OF DIAGONALIZABLE MATRICES
}

\author{
D. J. HARTFIEL
}

(Communicated by Lance W. Small)

ABSTRACT. This paper provides necessary and sufficient conditions for a subspace of matrices to contain a dense set of matrices having distinct eigenvalues.

A well-known and useful result in linear algebra is that matrices with distinct eigenvalues are dense in the set of $n \times n$ matrices. This result, however, does not hold for subspaces of matrices in general. For example, the subspace

$$
W=\left\{A: A=\left[\begin{array}{ll}
0 & 0 \\
a & 0
\end{array}\right] \quad \text { where } a \in R\right\}
$$

contains no matrix with distinct eigenvalues. In this paper we give necessary and sufficient conditions for a subspace of matrices to contain a dense set of matrices having distinct eigenvalues. The result is then applied to subspaces of matrices determined by specified 0 patterns.

\section{RESULTS}

All matrices in this paper are $n \times n$. We consider the real or the complex field. Throughout the paper we use that $W$ is a subspace of matrices. We let $W^{\prime}$ be the matrices in $W$ having distinct eigenvalues. We show that if $W$ contains a single matrix with distinct eigenvalues, then $W^{\prime}$ is dense in $W$.

Theorem 1. Let $W$ be a subspace of matrices. If $W^{\prime} \neq \varnothing$, then $W^{\prime}$ is dense in $W$.

Proof. Let $A \in W$. Choose any $B \in W^{\prime}$. Consider

$$
C(\alpha)=(1-\alpha) A+\alpha B \text { where } 0 \leq \alpha \leq 1 \text {. }
$$

Set

$$
\begin{aligned}
p_{\alpha}(x) & =\operatorname{det}(x I-C(\alpha)) \\
& =x^{n}+a_{1}(\alpha) x^{n-1}+\cdots+a_{n}(\alpha) .
\end{aligned}
$$

It is known [1, p. 81] that

$$
a_{k}(\alpha)=(-1)^{k} \sum_{i_{1}<i_{2}<\cdots<i_{m}} \Delta\left(i_{1}, i_{2}, \ldots, i_{m}\right)
$$

where $\Delta\left(i_{1}<i_{2}<\cdots<i_{m}\right)$ is the determinant of the submatrix of $C(\alpha)$ in rows and columns $i_{1}, \ldots, i_{m}$. Thus, each $a_{k}(\alpha)$ is a polynomial in $\alpha$.

Received by the editors October 7, 1993.

1991 Mathematics Subject Classification. Primary 15A18. 
It is easily seen that $p_{\alpha}$ has multiple roots if and only if $p_{\alpha}$ and $p_{\alpha}^{\prime}$ have a common root. Using that

$$
\begin{aligned}
& \operatorname{Res}\left(p_{\alpha}, p_{\alpha}^{\prime}\right)
\end{aligned}
$$

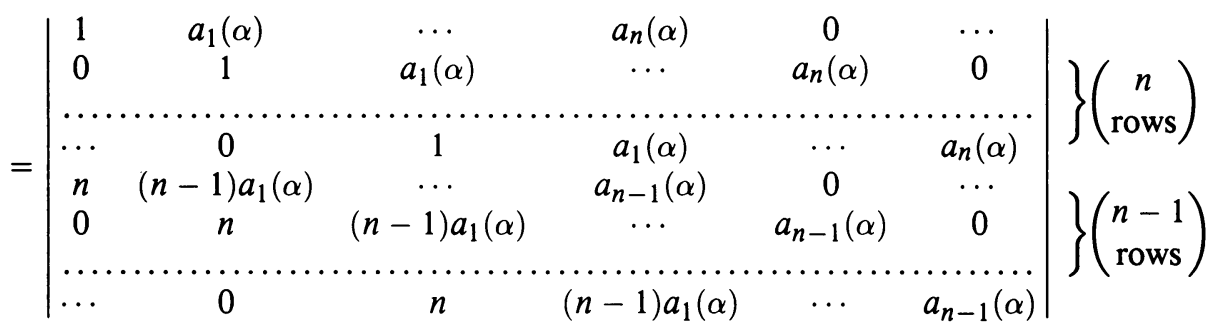

it follows from [4, p. 309] that $p_{\alpha}$ and $p_{\alpha}^{\prime}$ have common roots iff

$$
\operatorname{Res}\left(p_{\alpha}, p_{\alpha}^{\prime}\right)=0 \text {. }
$$

Now, since $\operatorname{Res}\left(p_{\alpha}, p_{\alpha}^{\prime}\right)$ is a polynomial in $\alpha$, either

(i) $\operatorname{Res}\left(p_{\alpha}, p_{\alpha}^{\prime}\right)=0$ for all $\alpha$ or

(ii) $\operatorname{Res}\left(p_{\alpha}, p_{\alpha}^{\prime}\right)=0$ for only finitely many $\alpha$.

Since $C(1)=B$, and $B$ has distinct eigenvalues, (i) can not occur. Thus (ii) occurs which means that for arbitrarily small $\alpha, \operatorname{Res}\left(p_{\alpha}, p_{\alpha}^{\prime}\right) \neq 0$ and so for such $\alpha, p_{\alpha}$ and $p_{\alpha}^{\prime}$ have no root in common. This means that, for such $\alpha, C(\alpha)=(1-\alpha) A+\alpha B$ has distinct eigenvalues and thus there is a matrix in $W$, namely such $C(\alpha)$, with distinct eigenvalues, arbitrarily close to $A$. From this, we see that $W^{\prime}$ is dense in $W$.

The proof also shows the following.

Corollary 1. Let $K$ be a convex set of matrices and $K^{\prime}$ those matrices in $K$ which have distinct eigenvalues. If $K^{\prime} \neq \varnothing$, then $K^{\prime}$ is dense in $K$.

The matrix

$$
C=\left[\begin{array}{cccccc}
0 & 1 & 0 & \cdots & 0 & 0 \\
0 & 0 & 1 & \cdots & 0 & 0 \\
\cdots & \ldots & \ldots & \ldots & \ldots & \ldots \\
0 & 0 & 0 & \cdots & 0 & 1 \\
1 & 0 & 0 & \cdots & 0 & 0
\end{array}\right]
$$

has eigenvalues $e^{i \frac{k 2 \pi}{n}}$ for $k=0, \ldots, n-1$. Thus, from the corollary, the set of stochastic matrices as well as the set of doubly stochastic matrices have dense sets consisting of matrices with distinct eigenvalues. These results were of importance in analyzing the behavior of a system in [3] and [6].

We now show how the theorem can be applied to a special subspace of matrices defined by patterns of 0 's. For this, we let $P$ be a $(0,1)$-matrix. By $P_{i i}$ we mean the matrix obtained from $P$ by deleting the $i$-th row and the $i$-th column.

Let $A$ be any matrix. Notationally, let

$$
A^{*}=\left(a_{i j}^{*}\right) \quad \text { where } a_{i j}^{*}= \begin{cases}1 & \text { if } a_{i j} \neq 0, \\ 0 & \text { otherwise. }\end{cases}
$$

The desired subspace is defined by

$$
W_{p}=\left\{A: A \text { is a matrix such that } A^{*} \leq P \text {, componentwise }\right\} .
$$


To describe when $W_{p}$ contains a dense set of matrices having distinct eigenvalues we use the following notion. A matrix $A$ has a diagonal if it has nonzero entries $a_{1 \sigma(1)}, a_{2 \sigma(2)}, \ldots, 2_{n \sigma(n)}$ where $\sigma$ is a permutation of $1,2, \ldots, n$. Diagonals in matrices have been studied in terms of their 0 patterns by numerous authors; for example, see [2], [5], and [7].

A lemma, which is easily proved, is helpful.

Lemma 1. Let $Q$ be a permutation matrix. Then there is a permutation matrix $R$ such that

$$
R Q R^{t}=C=\left[\begin{array}{cccc}
C_{1} & 0 & \cdots & 0 \\
0 & C_{2} & \cdots & 0 \\
\cdots & \ldots & \cdots & \ldots \\
0 & 0 & \cdots & C_{r}
\end{array}\right]
$$

where

$$
C_{i}=\left[\begin{array}{cccccc}
0 & 1 & 0 & \cdots & 0 & 0 \\
0 & 0 & 1 & \cdots & 0 & 0 \\
\cdots & \cdots & \cdots & \cdots & \cdots & \cdots \\
0 & 0 & 0 & \cdots & 1 & 0 \\
1 & 0 & 0 & \cdots & 0 & 0
\end{array}\right] .
$$

The desired theorem now follows.

Theorem 2. Let $P$ be a $(0,1)$-matrix. Then $W_{p}^{\prime}$ is dense in $W_{p}$ iff $P$ or $P_{i i}$ for some $i$ has a diagonal.

Proof. To argue the direct implication we will argue its contrapositive. Thus, suppose neither $P$ nor $P_{i i}$ for any $i$ has a diagonal. Let $A \in W_{p}$. Computing the coefficients of the characteristic polynomial of $A$ by using principle minors we see that since $a_{0}=a_{1}=0$,

$$
\operatorname{det}(x I-A)=x^{n}+a_{1} x^{n-1}+\cdots+a_{n-2} x^{2},
$$

so $x=0$ is an eigenvalue of $A$ of multiplicity at least two. Since $A$ was chosen arbitrarily, all matrices in $W_{p}$ have the eigenvalue 0 of multiplicity at least two and thus $W_{p}$ contains no matrix with distinct eigenvalues.

Conversely, suppose without loss of generality that $P$ or $P_{11}$ has a diagonal.

Case 1. Suppose $P_{11}$ has a diagonal. Using the lemma, we will also assume without loss of generality that the diagonal occupies the nonzero positions of $C$ in $P_{11}$. Thus,

$$
B=\left[\begin{array}{ll}
0 & 0 \\
0 & C
\end{array}\right] \leq P .
$$

Note that if $C_{k}$ is $n_{k} \times n_{k}$, then $C_{k}$ has eigenvalues $e^{i k \frac{2 \pi}{n_{k}}}$ for $k=1, \ldots, n_{k}$. Thus $C_{k}$ has distinct eigenvalues. Now,

$$
B^{\prime}=\left[\begin{array}{ccccc}
0 & 0 & 0 & \cdots & 0 \\
0 & C_{1} & 0 & \cdots & 0 \\
0 & 0 & 2 C_{2} & \cdots & 0 \\
\cdots & \ldots & \ldots \ldots \cdots \cdots & \ldots \ldots \\
0 & 0 & 0 & \cdots & r C_{r}
\end{array}\right] \in W_{p}
$$

and has distinct eigenvalues. Thus, by Theorem $1, W_{p}^{\prime}$ is dense in $W_{p}$. This yields the theorem. 
Case 2. If $P$ has a diagonal, then the argument of Case 1 can be adjusted to show that $W_{p}^{\prime} \neq \varnothing$ and hence again $W_{p}^{\prime}$ dense in $W_{p}$.

This yields the theorem.

In conclusion we can say that Theorem 1 may also be used in showing that other subspaces $W$ contain $W^{\prime}$ as a dense subset.

\section{REFERENCES}

1. Joel Franklin, Matrix theory, Prentice-Hall, Englewood Cliffs, NJ, 1968.

2. D. J. Hartfiel, A simplified form for nearly reducible and nearly decomposable matrices, Proc. Amer. Math. Soc. 24 (1970), 388-393.

3.

4. Nathan Jacobson, Basic algebra I, W. H. Freeman, San Francisco, CA, 1974.

5. Marvin Marcus and Henryk Minc, $A$ survey of matrix theory and matrix inequalities, Allyn and Bacon, Boston, MA, 1964.

6. Herbert Simon and Albert Ando, Aggregation of variables in dynamic systems, Econometrica 29 (1961), 111-138.

7. Richard Sinkhorn, Concerning a conjecture of Marshall Hall, Proc. Amer. Math. Soc. 21 (1969), 197-201.

Department of Mathematics, Texas A\&M University, College Station, TeXas 778433368

E-mail address: hartfiel@math. tamu.edu 